\title{
Mass spectrometry-based proteomics techniques and their application in ovarian cancer research
}

\author{
Agata Swiatly, Szymon Plewa, Jan Matysiak and Zenon J. Kokot ${ }^{*}$
}

\begin{abstract}
Ovarian cancer has emerged as one of the leading cause of gynecological malignancies. So far, the measurement of CA125 and HE4 concentrations in blood and transvaginal ultrasound examination are essential ovarian cancer diagnostic methods. However, their sensitivity and specificity are still not sufficient to detect disease at the early stage. Moreover, applied treatment may appear to be ineffective due to drug-resistance. Because of a high mortality rate of ovarian cancer, there is a pressing need to develop innovative strategies leading to a full understanding of complicated molecular pathways related to cancerogenesis. Recent studies have shown the great potential of clinical proteomics in the characterization of many diseases, including ovarian cancer. Therefore, in this review, we summarized achievements of proteomics in ovarian cancer management. Since the development of mass spectrometry has caused a breakthrough in systems biology, we decided to focus on studies based on this technique. According to PubMed engine, in the years 2008-2010 the number of studies concerning OC proteomics was increasing, and since 2010 it has reached a plateau. Proteomics as a rapidly evolving branch of science may be essential in novel biomarkers discovery, therapy decisions, progression predication, monitoring of drug response or resistance. Despite the fact that proteomics has many to offer, we also discussed some limitations occur in ovarian cancer studies. Main difficulties concern both complexity and heterogeneity of ovarian cancer and drawbacks of the mass spectrometry strategies. This review summarizes challenges, capabilities, and promises of the mass spectrometry-based proteomics techniques in ovarian cancer management.
\end{abstract}

Keywords: Ovarian cancer, Proteomics, Biomarkers, Drug-resistance, Diagnostics

\section{Background}

\section{Ovarian cancer}

Ovarian cancer (OC) causes about 125,000 deaths each year, which corresponds to over $4 \%$ of women cancer deaths worldwide $[1,2]$. Only $5-10 \%$ of the OC cases are hereditary $[3,4]$. OC tumors generally originate from other gynecological tissues than ovaries. Interestingly, tumors involves the ovary tissue secondarily [5]. However, despite several hypotheses of the OC origin, understanding its pathogenesis is still insufficient. Therefore, it has become a widely researched topic in the field of molecular sciences, which may influence modern medicine. Unfortunately, even though progress is made in prevention, development of novel tools for

* Correspondence: zkokot@ump.edu.pl

Department of Inorganic and Analytical Chemistry, Poznan University of

Medical Sciences, Grunwaldzka 6 Street, 60-780 Poznań, Poland early diagnosis and improvement of pharmacological therapies, the survival rate for OC remains poor. Patients often experience some symptoms but these are ignored or overlap with other ailments. Premalignant phase is difficult to recognize. A lack of sufficient screening options results in late detection. Despite successful surgery and appropriate treatment based on intravenous or intraperitoneal platinum- and taxane-based chemotherapy, diagnosis at advanced stages lowers 5-year survival rate to $27 \%$ [6]. This is caused by at least two factors: disease extension and biological differences in widely disseminated tumors [7]. Currently, less than $40 \%$ of all diagnosed OC cases are cured. However, if the diagnosis was made at the first stage of the disease, treatment could be limited to a surgical intervention alone [8].

(c) The Author(s). 2018 Open Access This article is distributed under the terms of the Creative Commons Attribution 4.0 International License (http://creativecommons.org/licenses/by/4.0/), which permits unrestricted use, distribution, and reproduction in any medium, provided you give appropriate credit to the original author(s) and the source, provide a link to the Creative Commons license, and indicate if changes were made. The Creative Commons Public Domain Dedication waiver (http://creativecommons.org/publicdomain/zero/1.0/) applies to the data made available in this article, unless otherwise stated. 


\section{Proteomics in cancer biomarker discovery}

The improvement in -omics sciences, genomics, proteomics, metabolomics, has opened a new research chapter, which is expected to develop novel tools for early diagnosis, treatment monitoring or population screening $[9,10]$. Fundamentally, cancerogenesis is associated with a genetic defect and epigenetic changes [11]. Many studies suggest that germline mutations in Breast Cancer Gene 1 (BRCA1) (17q21, chromosome 17: base pairs 43,044,294 to $43,125,482$ ) and Breast Cancer Gene 2 (BRCA2) (13q12.3, chromosome 13: base pairs $32,315,479$ to $32,399,671)$ are associated with a risk of breast and ovarian cancer [12]. Moreover, in epithelial OC some sporadic BRCA1 and BRCA2 mutations may occur, including BRCA1 hypermethylation [13]. Currently, it is thought that BRCA could be a useful prognostic marker only in combination with other biomarkers [14]. Since proteins are expressed by genes, and they are functional factors in phenotype characterization, the study of proteome profiles may yield information crucial for cancer research. Predictive markers could increase our understanding of molecular processes and pathological mechanisms, which is a dire need in modern medicine [15]. Sporadic molecular mutations that occur during abnormal cellular proliferation result in changes in protein secretion, modification or degradation. Therefore, in-depth proteomics analysis of various biosamples (e.g., serum, plasma, urine, tissues) obtained from cancer patients may facilitate the study of tumorigenesis, therapy monitoring, and development of novel targeted treatments. However, biomarker discovery might be challenging, bearing in mind that biomarker should improve currently used diagnostic methods, increase their sensitivity and specificity, provide optimal treatment, correspond to disease stage, and also be easily available in biofluids [16].

So far, proteomics methods have revealed thousands of potential cancer biomarkers. Most of the proteins suggested in the literature as clinically useful molecules are still awaiting proper validation. Hypothesis-testing is one of the most critical aspects of the cancer research. Another challenge in biomarker discovery is standardization and optimization of protocols. Some approaches are characterized by low precision and reproducibility, which is associated with poor study design [17]. Moreover, there are also biological challenges like sample variability or cancer heterogeneity. Nevertheless, a few biomarkers have been successfully implemented into clinical practice. To date, American Food and Drug Administration approved Cancer Antigen 125 (CA125) and human epididymis protein 4 (HE4) as circulating OC biomarkers for therapy monitoring and recurrence identification [18]. However, these tests have some inherent limitations, and their sensitivity and specificity should be increased, especially for patients with early stage of the tumor.It has been proved that combination of existing biomarkers with additional markers in one discriminatory model may improve their performance [19]. Therefore, it may be suggested that proteomics is a chance to develop novel tools that will significantly reduce the $\mathrm{OC}$ mortality rate.

\section{Mass spectrometry techniques in clinical proteomics}

The use of contemporary mass spectrometry (MS) represented a significant breakthrough in proteomics analysis. Since fast-evolving MS-techniques have a great impact on biomedical science, this innovative technology was introduced into clinical research [20]. Recently, matrix-assisted laser desorption/ionization (MALDI), and surface-enhanced laser desorption/ionization (SELDI) connected with timeto-flight (TOF) detector as well as electrospray ionization (ESI) have been extensively used in clinical proteomics [21-23]. Linking MS techniques with liquid chromatography (LC) or capillary electrophoresis allows for obtaining high resolution spectral proteomic patterns from numerous sample types [24-27]. These methods have already been reported as accurate tools for discovering multi-component classifiers, which significantly discriminate cancer samples from control biofluids or tissues. A crucial step in every analytical experiment is the choice of an optimal approach. Bearing in mind complexity of biological samples, the proper methodology should be chosen with respect to both sample pretreatment and MS-based strategy [28]. Initially, MS was used in clinical proteomics only to identify proteins and peptides. Today, the introduction of technical advances allows also for quantitative investigations.

This review presents proteomics strategies based on MS techniques used in OC research. We summarize successes of the proteomics in OC management and discuss challenges associated with biomarker discovery and proteome analysis. Moreover, we discuss improvements in MS strategies and prospects for effective diagnosis and treatment of OC. The use of proteomics and mass spectrometry in the ovarian cancer studies in the years 2008-2017 is presented in the Fig. 1. It was prepared based on PubMed engine (http://www.ncbi.nlm.nih.gov/ pubmed/) using the following keywords: "ovarian cancer and proteomics and mass spectrometry" and "ovarian cancer and proteomics". In the years 2008-2010 the number of studies concerning OC proteomics was increasing, and since 2010 it has been approximately at the same level. While, the contribution of the MS techniques in these studies is significant over the years.

\section{Characterization of proteins in the OC development}

The study of differentially expressed proteins in biosamples derived from OC patients or cell lines may improve early detection, treatment, and prognosis. MS-based 


\section{PROTEOMICS IN THE OVARIAN CANCER STUDIES IN THE YEARS 2008-2017}

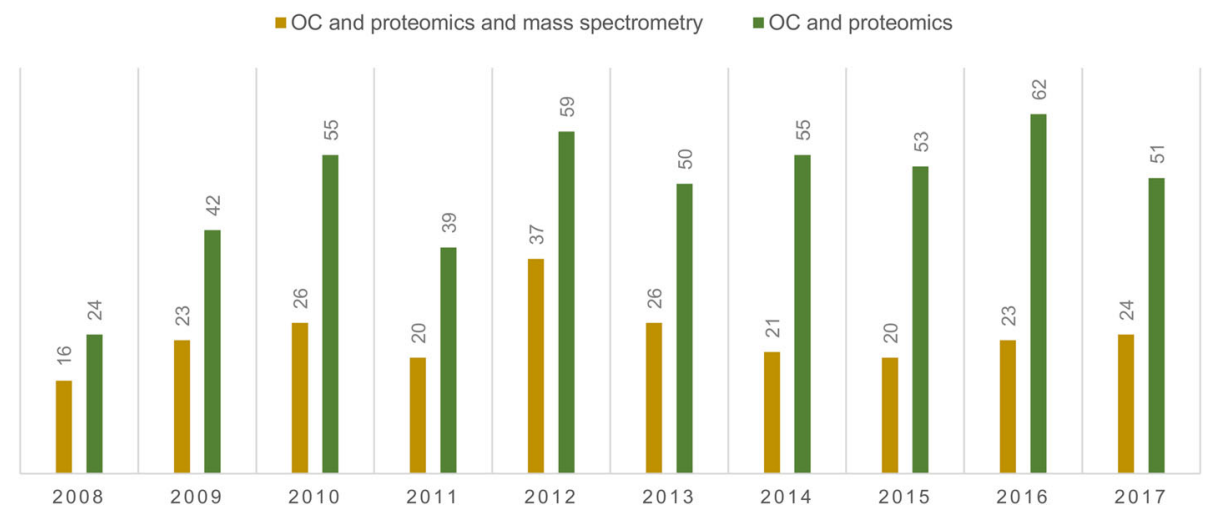

Fig. 1 The use of proteomics and mass spectrometry in the ovarian cancer studies, according to PubMed

proteomics techniques are widely employed to search sensitive and specific biomarkers, which, especially in combination with current diagnostic methods, may contribute to the detection of early stage OC. Moreover, identifying a relationship between overexpressed protein and dysfunctions in the cell cycle such as angiogenesis, apoptosis or proliferation, may be important for the development of novel therapies. Inhibition of the expression of significant proteins is a common method used to stop oncogenesis. Additionally, specific profiles of the proteins may indicate prognosis for the patients and help with proper treatment recommendations. Therefore, in this chapter, we discuss different MS-based strategies used in the clinical proteomics and their achievements in the field of the OC.

\section{Protein-peptide profiling}

Petricoin et al. were the first who introduced low-molecular-weight protein profiling into cancer proteomics [29]. Discovery and identification of proteomic patterns that reflect specific health conditions have become promising tools in clinical investigations. Both MALDI-TOF and SELDI-TOF MS are soft ionization techniques that cause minimal fragmentation of the obtained ions. Therefore, they are mainly used for protein profiling in low mass range. The resulting profiles may contain even thousands of data points - registered ions (mass/charge ratio - $\mathrm{m} / \mathrm{z}$ ), which are subjected to sophisticated data analysis. Bioinformatic tools select the most discriminative $\mathrm{m} / \mathrm{z}$ features, based on varying peaks intensities/areas, and define proteomic pattern characteristic for the study groups of samples. Subsequently, the differentiating peaks may be identified as proteins using tandem MS or protein databases. However, the identification process is omitted in some papers and the results are presented only as the $\mathrm{m} / \mathrm{z}$ values [16]. Protein-peptide profiling offers various advantages, like a discovery of multi-marker panels, which usually demonstrate a higher level of discriminatory information comparing with single markers, or quick analysis of large groups of samples [30].

Protein-peptide profiling studies involve inexpensive and minimally invasive procedure of collecting blood and its components like serum or plasma. Moreover, other blood tests, which determine important factors and provide information about general patient condition, can be routinely performed in clinical laboratories. Biofluids represent the overall pathophysiological status of a studied individual [31]. Therefore, serum and plasma protein-peptide profiling has become a popular strategy in the field of ovarian cancer. In 2002, Petricoin et al. used SELDI-TOF MS to analyze serum samples from ovarian cancer patients and control group that contained healthy individuals and women with benign tumors [29]. A combination of a genetic algorithm and cluster analysis resulted in the selection of five $\mathrm{m} / \mathrm{z}$ features: 534 , 989, 2111, 2251, and 2465. The obtained discriminative panel significantly differentiated the study groups (sensitivity of $100 \%$, specificity of $95 \%$, and positive predictive value of $94 \%$ ).

Further proteomic studies suggested that the use of a proper sample preparation method is required in order to detect aberrations in low abundance proteome. It is expected that potential biomarkers are mostly present at low concentrations, which disturbs their detection. For example, albumin, immunoglobulins, complement system proteins and transferrin are high-abundant blood proteins that represent about $99 \%$ of all serum proteins. Remaining $1 \%$ may be a rich source of yet unknown biomarkers [32]. To overcome the problem of high-abundance proteins, different strategies have been applied in OC biomarker discovery: immobilized metal affinity capture (IMAC) technology [33], immunodepletion [34], magnetic beads 
[35], solid-phase extraction [19], biomarker enrichment kits [30], and anion exchange chromatography [36]. Table 1 presents $\mathrm{m} / \mathrm{z}$ ratios and proteins identified as potential OC serum biomarkers. Despite application of purification techniques, most of the identified proteins are still highly abundant ones, such as apolipoprotein A1 or complement component 3. Moreover, these proteins seem to be unspecific, as they are also differently expressed in various types of cancer [37, 38]. However, the features of these proteins might be used to create multivariate predictive models, especially in combination with two common OC biomarkers - CA125 and HE4. Recently, a new OC diagnostic test was proposed - OVA 1, which is based on determination of serum levels of five different factors: CA125, apolipoprotein A1, beta-2-macroglobulin, transferrin, and transthyretin [39]. Therefore, protein-peptide profiling studies may be not a proper strategy to discover single useful biomarkers but they could contribute to developing multifactor tests.

\section{Quantitative proteomics}

Recently, new quantitative strategies of protein analysis have enjoyed huge attention. Since a discovery that proteomic changes under specific physiological conditions may help elucidate disease mechanisms and identify crucial

Table $1 \mathrm{M} / \mathrm{z}$ features (peptides) proposed as potential OC biomarkers in protein-peptide profiling studies

\begin{tabular}{lll}
\hline $\mathrm{m} / \mathrm{z}$ & Identified protein & Ref. \\
\hline $1082.24 ; 1087.80 ; 1066.08 ; 1277.19 ;$ & no identification & {$[34]$} \\
$1293.36 ; 1897.52 ; 4466.86 ; 4467.05 ;$ & & \\
$4469.14 ; 4962.52 ; 8601.58 ; 8601.64 ;$ & & \\
$11,693.29 ; 11,735.91 ; 17,105.23$ & & {$[35]$} \\
$5486 ; 6440 ; 13,720$ & & {$[36]$} \\
28,043 & apolipoprotein A1 & {$[36]$} \\
12,828 & transthyretin & {$[30]$} \\
2898.54 & & {$[19]$} \\
2210.80 & & {$[36]$} \\
3272 & inter-a-trypsin inhibitor & {$[30]$} \\
$2582.35 ; 3027.57$ & heavy chain H4 & {$[19]$} \\
$2082.73 ; 3158.75$ & & {$[30]$} \\
1041.68 & keratin 2a & {$[30]$} \\
1224.68 & glycosyltransferase-like 1B & {$[30]$} \\
$1690.94 ; 1777.97 ; 1865.01 ; 2021.11$ & complement component 3 & {$[19]$} \\
$1505.24 ; 2023.17$ & precursor & {$[30]$} \\
1739.93 & complement component & {$[30]$} \\
& 4A preproprotein & \\
1966.91 & casein kinase II alpha 1 & {$[30]$} \\
& subunit isoform a & \\
2115.05 & D-amino-acidoxidase & {$[30]$} \\
2345.19 & transgelin 2 & {$[30]$} \\
3239.55 & fibrinogen alpha chain & {$[30]$} \\
& isoform alpha & \\
1945.38 & preproprotein & \\
\hline & kininogen - 1 & {$[19]$} \\
\hline
\end{tabular}

biomarkers, a sensitive and accurate method for this purpose has been sought for. MS-based proteomics strategies may be divided into: "top-down" and "bottom-up" proteomics. In this review, we focus on the "bottom-up" strategy that is most common in clinical proteomics. The classical "bottom-up" strategy is based on staining proteins and two-dimensional electrophoresis. Stained spots of different abundance are digested, excised and identified mainly using MS techniques. Unfortunately, this strategy has many restrictions, as not all types of proteins are suitable for in-gel separation. Moreover, gel-to-gel variability made this method not significantly reproducible [40]. Due to these limitations, the classical approach seems to be often unreliable and quantitative analysis might be difficult to achieve. Therefore, the introduction of LC coupled with MS facilitated utilization of a novel "bottom-up" approach. A typical analysis starts with enzymatic (usually tryptic) digestion of the study proteins. Then the resulting short peptides are separated by LC, and eluates are further analyzed with tandem MS.

Recently two gel-free "bottom-up" strategies have been developed: isotopic labeling (like chemical isotopic labeling, isobaric tagging, metabolic isotopic labeling) and label-free analysis [41, 42]. In the label-based approach, peptides are linked with various tags in which ion signals correspond to relative peptide abundance in the analyzed sample. The most common labeling strategies include: SILAC (stable isotope labeling by amino acids in cell culture), ICAT (Isotope-coded affinity tag), iTRAQ (isobaric Tags for Relative and Absolute Quantification) and TMT (Tandem Mass Tags). Contrary to that, label-free analysis is based on precursor or spectral count. This approach has become popular due to a simpler sample preparation method and higher dynamic range as compared with labeling techniques [24]. The improvement of label-free approach consists in the advance of high-resolution mass spectrometer such as Orbitrap as well as TOF instruments [42].

Apart from the division into label-based and label-free methods presented in Fig. 2, two groups of "bottom-up" proteomics can be distinguished: discovery proteomics and targeted proteomics. The discovery proteomics is also called "shotgun" strategy, and it represents data-dependent acquisition. A considerable advantage of this method is a possibility to analyze thousands of proteins in one run. However, "shotgun" methods are usually characterized by low repeatability of peptide identification, which may be overcome by applying the targeted approach. Targeted proteomics techniques are mainly based on multiple-, selected- or parallel reaction monitoring, which results in sensitive and reproducible quantification of predefined proteins [42]. In the last few years, a third kind of acquisition has been developed: data-independent acquisition (DIA). During DIA analysis 


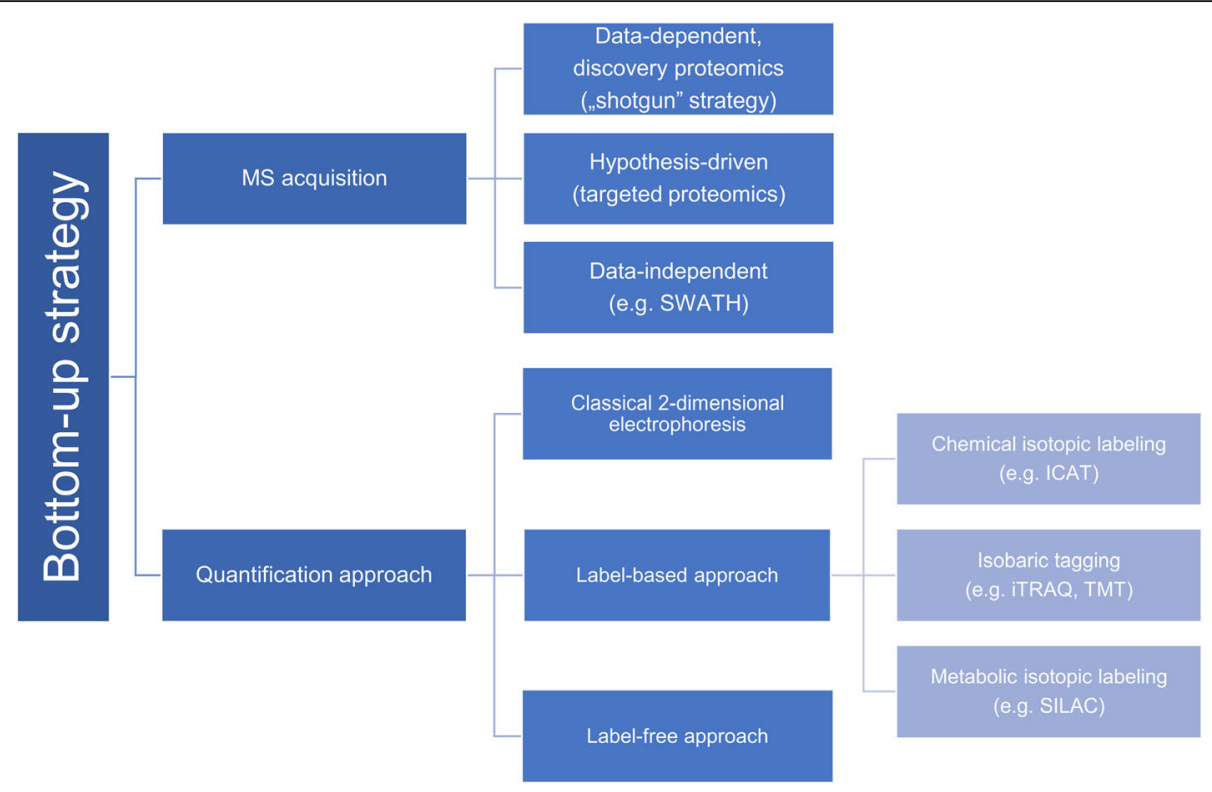

Fig. 2 Division of "bottom up" strategy according to quantification approach and MS acquisition

precursor ions from determined $\mathrm{m} / \mathrm{z}$ isolation window are deterministically fragmented. Recently, sequential window acquisition of all theoretical mass spectra (SWATH) (exemplified by DIA) has become a popular approach for biomarker discovery based on spectral libraries and targeted data analysis [43].

Many different quantitative strategies have been applied in the ovarian cancer proteomics. The most commonly performed analysis of ovarian cell lines are based on SILAC strategy. Isotope label is bound directly to the proteins during their synthesis. One of the first studies in this field compared the usefulness of classical two-dimensional electrophoresis coupled with SILAC strategy for describing urokinase plasminogen activator influence on $\mathrm{OC}$ cells. It proved that labeling technique is characterized by low quantitative variation. Moreover, this research demonstrated that urokinase plasminogen activator is capable of changing the expression of some proteins like thioredoxin, annexin IV, and fatty acid binding protein 3 in the OC cells [44]. Another study suggested that calcium-activated chloride channel regulator 1 and chloride channels may be potential OC therapeutic targets [45]. SILAC strategy was also used to investigate oxidative stress in the OC cells, which is an important issue in developing novel photodynamic therapy agents [46]. It was also revealed that epithelial-mesenchymal transition activated by epidermal growth factor modifies metabolic processes and cell cycle control. Therefore, it is associated with OC development and progression [47].

Another approach widely used in OC characterization is isobaric tagging: TMT and iTRAQ [48]. These techniques allow for simultaneous identification as well as relative quantification of proteins in multiple samples. Isobaric tagging patterns are mainly focused on an analysis of proteolytic digestion of proteins and peptides. ITRAQ labeling combined with tandem MS techniques enabled a selection of a few potential OC biomarkers: serum amyloid A-4 [49], astacin-like metalloendopeptidase [49], protein S100-A11, Keratin type II cytoskeletal 8, inorganic pyrophosphatase, isocitrate dehydrogenase [50], legumain [51], and protein Z [52]. Moreover, iTRAQ analysis was proved to be a useful method to track changes in proteins during a transition from benign to malignant tumor-like PI3K/Akt signaling pathway [53]. Another study aimed at comparing protein expression of ovarian cancer and endometrial high-grade serous carcinomas in both tissue samples and cell lines. Both tumors exhibited similar protein profiles [54]. TMT is much less popular in the field of OC proteome. Sinclair J. et al. combined this technique with two-dimensional electrophoresis, two-dimensional LC, and MS techniques: MALDI-TOF, electrospray quadrupole time-of-flight (Q-TOF) and Orbitrap to analyze proteome of two OC cell line models. A set of potential OC biomarkers was proposed for further examination. Additionally, the utility of the selected biomarker human active and pro-matrix metalloprotease-10, was examined using immunoassays to determine the level of this protein in human serum [55].

Although label-based strategies still remain the gold standard of quantitative proteomics, label-free methods have recently become more widespread. Therefore, LC combined with tandem MS was used to analyze plasma, ovary, and oviduct tissue samples from healthy and OC in chickens. The results were further compared to PCR 
and western blot analysis of human cell culture. Ovostatin 2 level was elevated in all the chicken samples as well as in human OC mRNA and cell lines [56]. The label-free analysis was also used in order to discover changes in protein expression and molecular/biological pathways in serum and tissues derived from malignant OC compared with benign tumors. Apolipoprotein AI and serotransferrin levels were reduced in both serum and tissue from patients with malignant $\mathrm{OC}$ tumor. Moreover, analysis of the interactome, which comprise the whole set of molecular interactions in a cell, and the pathways suggested a potential role of Poly(rC)-binding protein 1 in OC pathogenesis [57].

Although quantitative proteomics studies have significantly contributed to the discovery of $\mathrm{OC}$ protein patterns and cellular signaling networks, the approach has some limitations. SILAC method is mainly dedicated for cell line studies, which prevents human samples analysis [41]. The use of isobaric tagging patterns may be restricted by isotopic contaminations or background interference. Moreover, a common problem is co-isolation and fragmentation of some contamination ions together with the targeted precursor ion. Other drawbacks of the label-based approaches include high cost of the reagents and laborious sample preparation [16]. The major disadvantage of label-free methods is their low reproducibility. Additionally, they require extensive data processing and advanced statistical analysis [58].

\section{Post-translational modifications}

One of the challenges of the proteomic analysis is the proteome complexity, which results not only from high dynamic range of the biological samples but also from a wide variety of post-translational modifications e.g. glycosylation, ubiquitination, or phosphorylation. Many studies have proved that various types of cancers are associated with aberrations in protein modifications. Therefore, recent studies have focused on this field of proteomics to provide novel information on the disease development. In order to detect post-translational modifications and define their structures, sensitive MS methods are required like MALDI-TOF or LC-ESI (ESI - electrospray ionization) combined with dissociation techniques [59].

Protein glycosylation is undeniably a common and complex modification, responsible for several biological processes, like cell communication, signaling, adhesion, protein folding or solubility $[6,60]$. There are two main categories of glycosylation depending on glycan attachments: $\mathrm{N}$ - and $\mathrm{O}-$ glycans. Analysis and characterization of glycans may be challenging due to glycan variety and structure complexity [61]. To overcome this limitation, it is necessary to use targeted isolation and enrichment methods as well as sensitive detection techniques. Several methods have been proposed as glycoprotein affinity enrichment techniques: lectin chromatography, hydrazide chemistry, hydrophilic interaction liquid chromatography, or capture via immobilized titanium dioxide and boronic acid. Glycomics based on MS analysis has been successfully used to characterize the proteome of OC patients [60, 62]. A comparison of glycosyltransferases involved in N-linked pathway in $\mathrm{OC}$ and normal ovarian tissues allowed for identification of OC-specific glycoproteins and glycosylation aberrations. Selected glycoproteins in patient sera were verified using immunoprecipitation and microarray. Results pointed out periostin and thrombospondin as potential OC markers with cancer-specific glycosylation [63]. Furthermore, $\mathrm{N}$-linked sialylated glycoproteins were examined in OC and healthy controls sera. It was suggested that haptoglobin, PON1, and zinc-alpha-2-glycoprotein might have specific sialylation aberrations of the glycopeptides in the OC samples [64]. Glycomic strategies were also used to analyze proximal fluids derived from OC patients: ascites and malignant ovarian cyst fluids. Sialome (sialic acid that contains glycoproteins) of OC was identified, including a set of 13 sialoglycopeptides proposed as novel biomarkers [65]. The importance of the post-translational research was demonstrated in a study focused on CA125 $\mathrm{N}$-glycan forms. Differentiations between CA125 glycoforms from $\mathrm{OC}$ patients and controls may improve sensitivity and specificity of this widely used biomarker [66]. Recently, a novel methodology, coupling HILIC-UPLC and microarray for affinity-based study of cancer-associated glycans in OC cell lysates, was proposed. A structure of monoclonal A4 antibody was revealed. Understanding the antibody cancer-specific binding to glycans may simplify its use as a diagnostic indicator [67].

The intracellular signaling networks are mainly based on reversible protein phosphorylation. It is a highly dynamic post-translational modification responsible for e.g. metabolism, apoptosis, homeostasis or proliferation [68]. Phosphoproteins, like glycoproteins, are usually present in biological samples at low concentrations. Consequently, a number of enrichment strategies have been proposed: immunoprecipitation, immobilized metal affinity chromatography, metal-oxide affinity chromatography, chemical modification, magnetic beads or hydroxyapatite chromatography $[68,69]$. Recent studies have revealed the potential of phosphorylation profiling in oncogenesis characterization. Proteomic strategies have resulted in the discovery of targets of kinase inhibitors [70]. However, this approach is still out of favor according to $\mathrm{OC}$ studies. Research published by Francavilla et al. described phosphoproteomics as a promising strategy for understanding molecular determinants of OC. An analysis of epithelial cells collected from $\mathrm{OC}$ and healthy patients showed that cyclin-dependent kinase 7 (CDK7) controls cell 
proliferation. Therefore, inhibition of CDK7 may contribute to the development of efficient therapeutic strategies [71].

Although post-translational modification analysis seems to be an accurate and effective strategy for biomarker discovery and understanding cell signaling networking, there are some limitations that should be considered. Firstly, the modifications have to be subjected to chemical or enzymatic release. For example, ionization of native glycans is very poor [60]. Generally, phospho- and glyco-proteins are present in biosamples at low concentrations, so sufficient enrichment methods are required [72]. Moreover, reliable study results usually require derivatization. Sample preparation might be an issue mainly due to post-translational modification heterogeneity [73]. Finally, MS techniques have a limited dynamic range and minor modifications cannot be detected. Even though, when proper detection is achieved, accurate spectra interpretation and protein structure construction are challenging [72].

\section{Protein and peptide tissue imaging}

Human biofluids are usually characterized by high dynamic range, which complicates biomarker discovery process. As tissue samples are poor in proteins but rich in specific molecules related to disease, direct analysis of cancer tumors seems to be a promising approach. Standard tissue analysis is mainly based on immune-histochemistry (IHC) and histology. Nevertheless, deep proteomic tumor analysis may facilitate resolving the issues associated with $\mathrm{OC}$ diagnosis. The lack of significant biomarkers is not the only problem. Histological analysis usually confirms the disease and allows for tumor stage classification as per FIGO system. Further patient treatment depends on proper stage determination. However, staging is not sufficient as a prognostic parameter, and proper grade classification is an additional useful factor. Grading is assigned using light microscopy, which is rather subjective and not significantly reproducible. Therefore, new analytical methods are required to improve grading system and discover effective biomarkers. There are two rapidly evolving strategies to analyze proteins and peptides in tumor tissue: classical strategy and tissue imaging [74]. Classical proteomics such as LC-MS may be used to generate a molecular fingerprint of disease [75, 76]. These methods allow for identification of hundreds to thousands of proteins and peptides. However, the spatial distribution of specific molecules is lost in this kind of analysis as samples need to be homogenized. Recently, a direct MS analysis of tissue sections has become possible due to development of MALDI imaging mass spectrometry (MALDI-IMS) [77]. When coupled with histological analysis, MALDI-IMS may provide information not only about tissue proteome but also about the distribution of lipids, metabolites or xenobiotics. The use of MALDI technology requires a selection of matrix solution that coats tissue samples either with spray coating or with automatic matrix spotter. Firstly, MALDI-IMS was used to analyze fresh frozen tissue sections but this kind of material is usually not available during standard diagnostic setting. Thus, strategies focused on formalin fixed and paraffin embedded (FFPE) materials have been successfully adapted.

A number of MALDI-IMS analyses have been conducted in the field of OC. A comparison of MALDI profiling, MALDI-IMS, and IHC analysis enabled identification of Reg-Alpha Fragment of the 11S proteasome activator complex as a potential OC biomarker. The protein was present in OC tumor and absent in benign tissues [78]. Moreover, hierarchical cluster analysis was applied to prove that MALDI-IMS is effective in differentiation of tissue regions [79]. MALDI-IMS was also proved to be a useful strategy in the OC interface zone analysis. Plastin 2 and peroxiredoxin 1 were identified as upregulated proteins in tumor region as compared with a normal tissue [80]. Recently, a novel methodology focused on N-glycan analysis in FFPE OC tissue has been proposed. Imaging technology combined with ESI MS was used to generate images of $\mathrm{N}$-glycan structure distribution in tissue sections [81]. Another interesting approach is a combination of MALDI-IMS with top-down microproteomics. An analysis of benign, tumor and necrotic-fibrotic regions derived from OC biopsy specimens allowed for detection of promising novel biomarkers. The proposed methodology may contribute to identification of proteins that are usually lost during conventional proteomics analysis [82].

MALDI-IMS is potentially a revolutionary technique in pathology. Recently, tremendous improvement has been made in the field of sample preparation and instrumentation. However, the technique still poses a few challenges. First, there are no studies confirming its utility in clinical settings. Second, sample preparation and measurement methodology should be proved to be reproducible and robust in different laboratories. For better understanding of MALDI-IMS results, the integration with other -omics studies like transcriptomics still needs improvement $[77,79]$.

\section{Proteomics in treatment response}

Cytoreductive surgery coupled with chemotherapy based on paclitaxel, carboplatin, cisplatin, often in combination with taxanes, is the most common OC treatment option. A decision on postoperative chemotherapy depends on the risk of the tumor recurrence, stage, and grade. OC subtypes respond differently to treatment and chemo-resistance may occur as a serious side effect $[6,83]$. According to statistics, even $25 \%$ of patients experience platinum resistance, and in $50-60 \%$ of cases cancer resistance develops during the treatment [84]. Investigation of chemotherapy mechanisms and discovery of factors responsible for chemotherapy response and resistance provide a selection of alternative 
therapies or chemo-sensitizing agents [85]. Unfortunately, individual agents offer rather low response rate: $5-20 \%$. As a result, patients often have to choose between continuation of chemotherapy or supportive care only [84]. Drug resistance may occur due to pharmacokinetic, tumor-specific aberrations and microenvironment of the tumor [86]. Discovery of chemo-resistant biomarkers may improve personalized medicine and therapy planning. Therefore, a number of previously discussed techniques: MALDI-TOF MS, LC-MS/MS, SILAC, iTRAQ, label-free quantitation, ICAT, were proposed to identify new biomarkers and investigate proteome changes during $\mathrm{OC}$ treatment [85]. Previously identified chemo-resistance biomarkers are presented in Table 2.

MALDI-TOF MS strategy was first used to identify chemo-resistant biomarkers in a comparative analysis of platinum-sensitive and platinum-resistant cell lines. Five differentially expressed proteins were identified and further validated [87]. Another study focused on the same cell culture analysis discovered five new mitochondrial proteins potentially involved in chemo-resistance mechanisms. It was suggested that mitochondrial defects may be associated with drug resistance [88]. MALDI-TOF MS combined with LC-MS/MS analysis demonstrated that proteins responsible for metabolism, stress response, and apoptosis, are differentially expressed in paclitaxel sensitive and resistant OC cell lines. Moreover, one of disulfide isomerases was found to play an essential role in chemo-resistance [89]. Lee et al. also used a combination of two techniques: MALDI-TOF MS and LC-MS/MS and identified two potential chemo-resistant biomarkers in OC cells [90]. As glycosylation is often connected with cancer development, analysis of glycoproteins was used to discover useful biomarkers. Abnormalities in the expression of four glycoproteins were found to be characteristic of chemo-resistance cases [91].

A study utilizing label-free LC-MS/MS strategy confirmed that mitochondrial proteomic changes are important in both platinum [88] and cisplatin resistance [92]. Therefore, abnormalities in the concentration of three proteins were found to be characteristic of cisplatin-resistant OC cell line [92]. Moreover, the label-free approach confirmed that elevated levels of TXNDC17 in both OC cell and tissues are associated with paclitaxel resistance. The study additionally demonstrated the relationship between TXNDC17, poor prognostic factors, and short survival rate [93].

Recently, isotopic labeling has also been widely used in the investigation of drug resistance. SILAC analysis proved that, as in the case of platinum [88] and cisplatin resistance [92], doxorubicin resistance is partially caused by changes in mitochondria [94]. ICAT technique was applied in the study of cisplatin resistance. Changes in protein expression in chemo-resistant cells were compared with mRNA expression levels [95]. Furthermore, another study based on ICAT-MS/MS analysis integrated with RNA analysis pointed out 16 protein changes in the chemo-resistant $\mathrm{OC}$ tissues. These results triggered a conclusion that chemotherapy response and resistance are determined by a set of proteins from following classes: extracellular-matrix, junction or cell adhesion proteins [96]. ITRAQ strategy turned out to be a useful approach in multidrug-resistance investigations [97]. Moreover, iTRAQ technique revealed that major histocompatibility complex class I peptide repertoire of $\mathrm{OC}$ cells is associated with the pathological condition of the cell and may become a new treatment target also in chemo-resistant cancers [98]. Advanced bioinformatics approach contributed to establishing prediction models based on iTRAQ analysis results. It was proved that proteomic profiles of OC may provide information on platinum drug responses [99].

The studies on the chemoresistance mechanism are very promising and may improve the treatment and prognosis of patients with OC. However, there is still a lot of work ahead [86]. Firstly, many analyses are conducted only on cell lines. An appropriate clinical material is limited and difficult to obtain [85]. Therefore, all encouraging results should be verified using OC tissue. Despite high-throughput technologies, there is still no significant biomarker to predict a treatment response [85]. Moreover, many cancers may develop resistance to different drugs and therapies [6].

\section{Challenges for biomarker research in clinical proteomics}

Although clinical proteomics did contribute to understanding of complicated molecular pathways of several diseases including $\mathrm{OC}$, only few information and findings are significant enough to be translated into clinical settings. A great effort was made to introduce new, sensitive biomarkers to clinical practice. However, most of them did not meet the validation requirements. Currently, there seem to be a great number of studies focused on searching for marker candidates. Over the past decades, clinical proteomics has encountered some obstacles and challenges, which should be overcome in the future. In this chapter, we discussed the general challenges associated with the MS-based analysis of biomarkers. Drawbacks characteristic for the specific methods are presented in the particular chapters.

The first issue to deal with at the beginning of the research is experimental design. The sample size is often inadequate to draw any meaningful conclusions and to obtain significant statistical power. Selection and building of suitable statistical methods and models is an essential part of the experiment. Moreover, in order to ensure the necessary impact of the analysis, the study groups should be properly selected. Factors such as age, 
Table 2 Drug-resistance markers in OC identified by MS-based proteomic techniques

\begin{tabular}{|c|c|c|}
\hline Chemoresistance markers & MS technique & Ref. \\
\hline $\begin{array}{l}\text { Annexin 3; } \\
\text { Destrin; } \\
\text { Cofilin1; } \\
\text { Gluthathione-S transferase omega 1; } \\
\text { Cytosolic NADP+ dependent isocitrate dehydrogenase }\end{array}$ & MALDI-TOF & {$[87]$} \\
\hline $\begin{array}{l}\text { ATP synthase subunit alpha; } \\
\text { Peroxiredoxin 3; } \\
\text { Prohibitin; } \\
\text { Electron transfer flavoprotein subunit alpha; } \\
\text { Aldehyde dehydrogenase X }\end{array}$ & MALDI-TOF & {$[88]$} \\
\hline ERp57 & $\begin{array}{l}\text { MALDI-TOF } \\
\text { ESI-Q-TOF }\end{array}$ & [89] \\
\hline $\begin{array}{l}\text { Tumor rejection antigen (gp96) 1; } \\
\text { Triose phosphate isomerase; } \\
\text { Palmitoyl-protein thioesterase1 precursor; } \\
\text { ER-associated DNAJ }\end{array}$ & $\begin{array}{l}\text { MALDI-TOF } \\
\text { ESI-Q-TOF }\end{array}$ & [91] \\
\hline $\begin{array}{l}\text { Aldehyde dehydrogenase } 1 \text { family, member A1; } \\
\text { Annexin A1; } \\
\text { Heterogeneous Nuclear Ribonucleoproteins A2; } \\
\text { Rho GDP dissociation inhibitor }\end{array}$ & $\begin{array}{l}\text { MALDI-TOF } \\
\text { LC-MS/MS }\end{array}$ & [90] \\
\hline $\begin{array}{l}\text { Activated leukocyte cell adhesion molecule; } \\
\text { A kinase anchoring protein 12; } \\
\text { Nestin }\end{array}$ & Orbitrap & [92] \\
\hline Thioredoxin domain containing 17 & Orbitrap & [93] \\
\hline Mitochondrial topoisomerase I & Orbitrap & [94] \\
\hline $\begin{array}{l}\text { Cell recognition molecule CASPR3; } \\
\text { S100 protein family members; } \\
\text { Junction adhesion molecule Claudin 4; } \\
\text { CDC42-binding protein kinase beta }\end{array}$ & ESI-MS/MS & [95] \\
\hline $\begin{array}{l}\text { P53 binding protein } 1 ; \\
\text { Catenin delta } 1 \text { and plakoglobin; } \\
\text { EGF-containing fibulin-like extracellular matrix protein 1; } \\
\text { Voltage-dependent anion-selective channel protein } 1\end{array}$ & HPLC-ESI-MS/MS & [96] \\
\hline $\begin{array}{l}\text { Pyruvate kinase isozymes M1/M2; } \\
\text { Heat Shock Protein Family D }\end{array}$ & ESI-Q-TOF & [97] \\
\hline
\end{tabular}

diet, medication, etc. may have a crucial influence on the experiment [100]. Discovery of a sensitive and specific OC biomarker requires analysis and comparisons of several groups of samples including healthy controls, inflammatory controls, benign adnexal masses, early OC stage, and preferably other malignancies than OC. Proteins selected as relevant $\mathrm{OC}$ markers should be further compared with CA125 and HE4, and their utility as OC markers should be confirmed in longitudinal monitoring. Finally, for proper assessment of sensitivity and specificity, the selected markers need to be applied to blinded large study sets. Most of the experiments include only pilot analysis and omits another important step - expanding the study groups and sample size.

Another crucial factor in every proteomic experiment is the quality of clinical samples quality. The best way to collect samples is to create a biobank with all epidemiologic and genetic data. Samples should be collected in the conditions compatible with MS method and immediately frozen (e.g., in liquid nitrogen), preferably with the addition of protease inhibitors. One of the main pitfalls is the influence of inappropriate storage temperature and freeze-thaw cycles on the protein degradation. Endogenous contaminations with salts or lipids, and even inappropriate type of sample tube may affect the result of the experiment [101]. Therefore, in order to obtain the highest sample quality, a good communication between proteome scientists and clinical staff is necessary and all aspects of samples collection should be discussed.

Over the past years, unsuccessful efforts have been undertaken to discover OC screening method, including a unique proteomic signature. One of the main reasons for ineffective investigations is the limitations of MS methods. Despite significant progress in MS techniques, high dynamic proteome range still occurs as a major challenge. The most relevant proteins are usually present in biological samples in low concentrations, and their detection remains very difficult when high abundant proteins coexist. The most frequently used types of sample are serum and plasma. Since ten high abundant 
serum/plasma proteins account for about $90 \%$ of the total proteins, examination of all low concentrated proteins - the promising source of biomarkers is a huge challenge. To reduce the overall sample complexity, several methods have been proposed, including pre-fractionation, depletion or enrichment. However, proteomic techniques are still not sufficient to detect low abundant molecules. Although they are able to simplify the protein mixture, major blood components remain highly concentrated. Moreover, some depletion methods and other preanalytical steps lead to the removal of relevant proteins due to non-specific-bounds [16]. Therefore, the issue of low-abundance proteins analysis should be studied in the near future.

Even though MS-based techniques have been used in proteome science for a long time, there are still no uniform workflows and procedures. Therefore, inter-laboratory variability and low reproducibility often occur [16]. Another analytical challenge is statistical analysis and interpretation of the results containing hundreds, sometimes even thousands, of peptides and proteins. A typical result of the proteomic experiment is a list of interferences containing peptide-spectrum matches. Each interference is characterized by a score, which shows the confidence of correct identification. However, to avoid false-positive results, significant bioinformatics strategy may be a crucial step. $P$-value is not suitable for multiple testing of a database search, and need to be improved, usually by false discovery rate calculations [102].
Moreover, multiple comparisons require the use of proper corrections (e.g., Bonferroni correction, determination of the q-value) [72].

Past decade of MS-based research revealed many obstacles associated with biomarker discovery. Thus, more restrictions are required for each further analysis. Every experiment data should be validated with other complementary method like ELISA or western blot. Further analysis need to be also conducted using an external set of samples to ensure the reliability of the obtained results. Moreover, presented studies should be no longer limited to the list of differentially expressed proteins and peptides. Identified molecules need to be connected into one network and they need to explain complicated disease mechanisms. These steps are necessary for the future implementation of biomarker assessment to clinical practice.

Despite many challenges in biomarker studies, there are already two multimarker tests: OVA 1 and ROMA (risk of ovarian malignancy algorithm) approved by the Food and Drug Administration for OC diagnosis. Therefore, it should be expected that the current studies constitute a solid basis for creating new reliable diagnostic tools in the future. The addition of sophisticated MS techniques, like MALDI-IMS, to methods commonly used in the clinics, may be a step forward to significantly improve diagnosis and treatment.

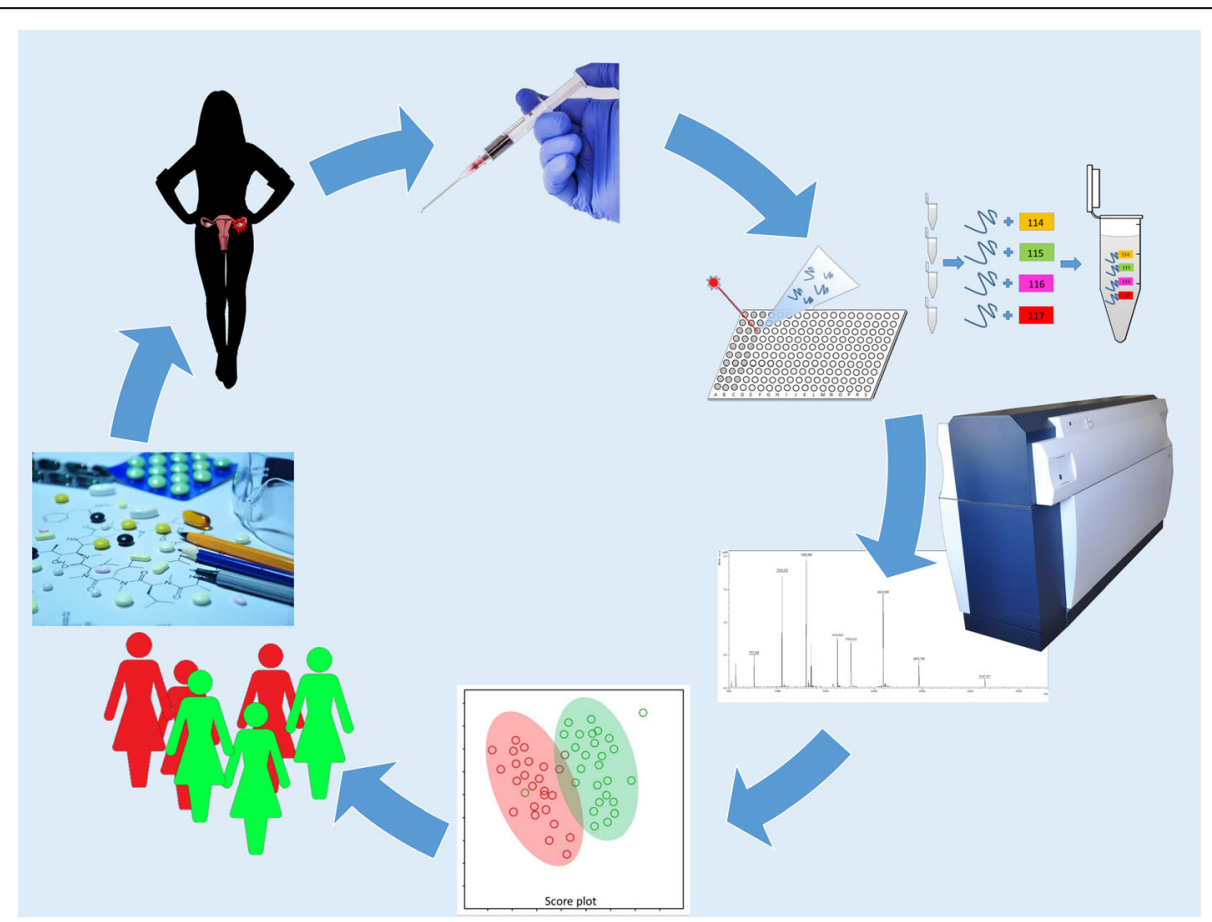

Fig. 3 Development of novel, innovative diagnostics methods, therapies or drug response monitoring based on proteomics techniques 


\section{Conclusions}

Since OC is responsible for thousands of deaths each year, there is a dire need to improve diagnostics as well as treatments strategies. Elucidating the mechanisms and molecular pathways during carcinogenesis may contribute to developing novel targeted therapies and personalized medicine. Proteomics, as a rapidly evolving branch of science, may be essential in novel biomarkers discovery, therapy decisions, progression prediction, and monitoring of drug response or resistance. This review presents main proteomics techniques based on MS that provide information on molecular mechanisms of the OC. Many limitations of particular approaches have also been discussed. The first challenge is the heterogeneity and diverse origins of the OC. It should be taken into account during basic and clinical studies design. Another difficulty is a limited access to biological samples, especially OC tissues. Studies based on the OC cell lines should be verified using tumor tissues. The next issue that should be considered is low inter-laboratory reproducibility of the results. There are many studies proposing different $\mathrm{OC}$ biomarkers but usually none further research is conducted to confirm the proposals. Moreover, there are still some improvements necessary in MS techniques. Analytical protocols need to be standardized to introduce a reproducible and high-throughput analysis. Lack of uniform workflow leads to false-positive results associated for example with sample collection errors. Moreover, high dynamic range of compounds in biological samples is an issue that is still not solved. A great challenge for proteome scientists is also developing significant bioinformatics tools and proper statistics strategies. Another important obstacle, frequently ignored, is validation of the obtained results and data interpretation that lead to understanding of complicated molecular pathways connected with the disease. However, current studies are getting more and more sophisticated. Due to multidisciplinary teams and close cooperation of clinical staff and scientists, the study design becomes more advanced. Authors believe that considering fast development of MS techniques, all drawbacks may be overcome in the next few years. Although the proteomics strategies still require optimization, they have also a lot to offer and in the near future they may provide insights into complicated and inaccessible cancer proteomics, which is presented in the Fig. 3.

\footnotetext{
Abbreviations

BRCA 1: Breast Cancer Gene 1; BRCA 2: Breast Cancer Gene 2; CA125: Cancer Antigen 125; CDK7: cyclin-dependent kinase 7; DIA: data-independent acquisition; ESI: electrospray ionization; FFPE: formalin fixed and paraffin embedded; HE4: human epididymis protein 4; ICAT: Isotope-coded affinity tag; IHC: immune-histochemistry; IMAC: immobilized metal affinity capture; IMS: MALDI imaging mass spectrometry; iTRAQ: isobaric Tags for Relative and Absolute Quantification; LC: liquid chromatography; m/z: mass/charge ratio; MALDI: matrix assisted laser desorption/ionization; MS: mass spectrometry;
}

OC: ovarian cancer; Q-TOF: quadrupole time-of-flight; ROMA: risk of ovarian malignancy algorithm; SELDI: surface-enhanced laser desorption/ionization; SILAC: stable isotope labeling by amino acids in cell culture; SWATH: sequential window acquisition of all theoretical mass spectra; TMT: Tandem Mass Tags; TOF: time-to-flight

\section{Funding}

The project was supported by the Polish National Science Centre (2014/15/ B/NZ7/00964)

\section{Authors' contributions}

A.S. and S.P. executed and drafted the manuscript. J.M. and Z.J.K made critical discussion about the study and designed structure of the manuscript. All authors have read and approved the manuscript submitted.

Ethics approval and consent to participate

Not applicable (review article)

Competing interests

The authors declare that they have no competing interests.

\section{Publisher's Note}

Springer Nature remains neutral with regard to jurisdictional claims in published maps and institutional affiliations.

Received: 19 April 2018 Accepted: 20 September 2018

Published online: 01 October 2018

\section{References}

1. Zhang B, Barekati Z, Kohler C, Radpour R, Asadollahi R, Holzgreve W, et al. Proteomics and biomarkers for ovarian cancer diagnosis. Ann Clin Lab Sci. 2010:40:218-25.

2. Poersch A, Grassi ML, Carvalho VP, Lanfredi GP, Palma Cde S, Greene LJ, et al. A proteomic signature of ovarian cancer tumor fluid identified by highthroughput and verified by targeted proteomics. J Proteomics. 2016;145:226-36.

3. Bast Jr RC, Hennessy B, Mills GB. The biology of ovarian cancer: new opportunities for translation.

4. Longuespée R, Boyon C, Desmons A, Vinatier D, Leblanc E, Farré I, et al. Ovarian cancer molecular pathology. Cancer Metastasis Rev. 2012;31:713-32

5. Reid BM, Permuth JB, Sellers TA. Epidemiology of ovarian cancer: a review. Cancer Biol Med. 2017;14:9-32.

6. Elzek MA, Rodland KD. Proteomics of ovarian cancer: functional insights and clinical applications. Cancer Metastasis Rev. 2015;34:83-96.

7. Vaughan S, Coward JI, Bast RC, Berchuck A, Berek JS, Brenton JD, et al. Rethinking ovarian cancer: recommendations for improving outcomes. Nat Rev Cancer. 2011:11:719-25.

8. Hays JL, Kim G, Giuroiu I, Kohn EC. Proteomics and ovarian cancer: integrating proteomics information into clinical care. J Proteome. 2010;73: 1864-72.

9. Parker $\mathrm{CE}$, Borchers $\mathrm{CH}$. Mass spectrometry based biomarker discovery, verification, and validation - quality assurance and control of protein biomarker assays. Mol Oncol. 2014;8:840-58.

10. Matthews H, Hanison J, Nirmalan N. "Omics"-informed drug and biomarker discovery: opportunities. Challenges and Future Perspectives Proteomes. 2016;4:28

11. Herceg Z, Hainaut P. Genetic and epigenetic alterations as biomarkers for cancer detection, diagnosis and prognosis. Mol Oncol. 2007:1:26-41.

12. Rebbeck TR, Mitra N, Wan F, Sinilnikova OM, Healey S, McGuffog L, et al. Association of type and location of BRCA1 and BRCA2 mutations with risk of breast and ovarian cancer. JAMA. 2015;313:1347-61.

13. Gee ME, Faraahi Z, McCormick A, Edmondson RJ. DNA damage repair in ovarian cancer: unlocking the heterogeneity. J Ovarian Res. 2018;11:50

14. Ardekani AM, Liotta LA, Petricoin EF. Clinical potential of proteomics in the diagnosis of ovarian cancer. Expert Rev Mol Diagn. 2002;2:312-20.

15. Sajic T, Liu Y, Aebersold R. Using data-independent, high resolution mass spectrometry in protein biomarker research: perspectives and clinical applications. Proteomics Clin Appl. 2014.

16. Hajduk J, Matysiak J, Kokot ZJ. Challenges in biomarker discovery with MALDI-TOF MS. Clin Chim Acta. 2016. 
17. Meehan KL, Rainczuk A, Salamonsen LA, Stephens AN. Proteomics and the search for biomarkers of female reproductive diseases. Reproduction. 2010; 140:505-19.

18. Romagnolo C, Leon AE, Fabricio ASC, Taborelli M, Polesel J, Del Pup L, et al. HE4, CA125 and risk of ovarian malignancy algorithm (ROMA) as diagnostic tools for ovarian cancer in patients with a pelvic mass: an Italian multicenter study. Gynecol Oncol. 2016;141:303-11.

19. Swiatly A, Horala A, Hajduk J, Matysiak J, Nowak-Markwitz E, Kokot ZJ. MALDI-TOF-MS analysis in discovery and identification of serum proteomic patterns of ovarian cancer. BMC Cancer. 2017;17.

20. Imperlini E, Santorelli L, Orrù S, Scolamiero E, Ruoppolo M, Caterino M. Mass spectrometry-based Metabolomic and proteomic strategies in organic Acidemias. Biomed Res Int. 2016;2016:9210408

21. Merlos Rodrigo MA, Zitka O, Krizkova S, Moulick A, Adam V, Kizek R. MALDITOF MS as evolving cancer diagnostic tool: a review. J Pharm Biomed Anal. 2014;95:245-55

22. Tabb DL. Quality assessment for clinical proteomics. Clin Biochem. 2013; 46:411-20.

23. Albalat A, Husi H, Stalmach A, Schanstra JP, Mischak H. Classical MALDI-MS versus CE-based ESI-MS proteomic profiling in urine for clinical applications. Bioanalysis. 2014:6:247-66.

24. Sandin M, Chawade A, Levander F. Is label-free LC-MS/MS ready for biomarker discovery? PROTEOMICS - Clin Appl. 2015;9:289-94.

25. Collins MA, An J, Hood BL, Conrads TP, Bowser RP. Label-free LC-MS/ MS proteomic analysis of cerebrospinal fluid identifies protein/pathway alterations and candidate biomarkers for amyotrophic lateral sclerosis. J Proteome Res. 2015;14:4486-501.

26. Tsai T-H, Song E, Zhu R, Di Poto C, Wang M, Luo Y, et al. LC-MS/MS-based serum proteomics for identification of candidate biomarkers for hepatocellular carcinoma. Proteomics. 2015;15:2369-81.

27. Stalmach A, Husi H, Mosbahi K, Albalat A, Mullen W, Mischak H. Methods in Capillary Electrophoresis Coupled to Mass Spectrometry for the Identification of Clinical Proteomic/Peptidomic Biomarkers in Biofluids. 2015. p. 187-205.

28. Dittrich J, Becker S, Hecht M, Ceglarek U. Sample preparation strategies for targeted proteomics via proteotypic peptides in human blood using liquid chromatography tandem mass spectrometry. PROTEOMICS - Clin Appl. 2015;9:5-16.

29. Petricoin EF, Ardekani AM, Hitt BA, Levine PJ, Fusaro VA, Steinberg SM, et al. Use of proteomic patterns in serum to identify ovarian cancer. Lancet. 2002; 359:572-7.

30. Lopez MF, Mikulskis A, Kuzdzal S, Golenko E, Petricoin EF, Liotta LA, et al. A novel, high-throughput workflow for discovery and identification of serum carrier protein-bound peptide biomarker candidates in ovarian cancer samples. Clin Chem. 2007;53:1067-74.

31. Ye H, Sun L, Huang X, Zhang P, Zhao X. A proteomic approach for plasma biomarker discovery with 8-plex iTRAQ labeling and SCX-LC-MS/MS. Mol Cell Biochem. 2010;343:91-9.

32. Aresta A, Calvano CD, Palmisano F, Zambonin CG, Monaco A, Tommasi $S$, et al. Impact of sample preparation in peptide/protein profiling in human serum by MALDI-TOF mass spectrometry. J Pharm Biomed Anal. 2008;46:157-64.

33. Ye B, Cramer DW, Skates SJ, Gygi SP, Pratomo V, Fu L, et al. Haptoglobin-a subunit as potential serum biomarker in ovarian Cancer. Clin Cancer Res. 2003:9.

34. Periyasamy A, Gopisetty G, Veluswami S, Joyimallaya Subramanium M, Thangarajan R. Identification of candidate biomarker mass ( $\mathrm{m} / \mathrm{z}$ ) ranges in serous ovarian adenocarcinoma using matrix-assisted laser desorption/ionization timeof-flight mass spectrometry profiling. Biomarkers. 2015;20:292-8.

35. Wu S, Xu K, Chen G, Zhang J, Liu Z, Xie X. Identification of serum biomarkers for ovarian cancer using MALDI-TOF-MS combined with magnetic beads. Int J Clin Oncol. 2012;17:89-95.

36. Zhang Z, Bast RC, Yu Y, Li J, Sokoll L, Rai AJ, et al. Three biomarkers identified from serum proteomic analysis for the detection of early stage ovarian cancer. Cancer Res. 2004;64:5882-90.

37. Li C, Li H, Zhang T, Li J, Liu L, Chang J. Discovery of Apo-A1 as a potential bladder cancer biomarker by urine proteomics and analysis. Biochem Biophys Res Commun. 2014;446:1047-52.

38. Kawahara R, Bollinger JG, Rivera C, Ribeiro ACP, Brandão TB, Leme AFP, et al. A targeted proteomic strategy for the measurement of oral cancer candidate biomarkers in human saliva. Proteomics. 2016;16:159-73.
39. Grenache DG, Heichman KA, Werner TL, Vucetic Z. Clinical performance of two multi-marker blood tests for predicting malignancy in women with an adnexal mass. Clin Chim Acta. 2015;438:358-63.

40. Bland AM, D'Eugenio LR, Dugan MA, Janech MG, Almeida JS, Zile MR, et al. Comparison of variability associated with sample preparation in two-dimensional gel electrophoresis of cardiac tissue. J Biomol Tech. 2006;17:195-9.

41. Lindemann C, Thomanek N, Hundt F, Lerari T, Meyer HE, Wolters D, et al. Strategies in relative and absolute quantitative mass spectrometry based proteomics. Biol Chem. 2017;398:687-99.

42. Schubert OT, Röst HL, Collins BC, Rosenberger G, Aebersold R. Quantitative proteomics: challenges and opportunities in basic and applied research. Nat Protoc. 2017:12:1289-94.

43. Collins BC, Hunter CL, Liu Y, Schilling B, Rosenberger G, Bader SL, et al. Multi-laboratory assessment of reproducibility, qualitative and quantitative performance of SWATH-mass spectrometry. Nat Commun. 2017;8:291.

44. Uitto PM, Lance BK, Wood GR, Sherman J, Baker MS, Molloy MP. Comparing SILAC and two-dimensional gel electrophoresis image analysis for profiling Urokinase plasminogen activator signaling in ovarian Cancer cells. J Proteome Res. 2007:6:2105-12.

45. Musrap N, Tuccitto A, Karagiannis GS, Saraon P, Batruch I, Diamandis EP. Comparative proteomics of ovarian Cancer aggregate formation reveals an increased expression of calcium-activated Chloride Channel regulator 1 (CLCA1). J Biol Chem. 2015;290:17218-27.

46. Qi D, Wang Q, Li H, Zhang T, Lan R, Kwong DWJ, et al. SILAC-based quantitative proteomics identified lysosome as a fast response target to PDT agent Gd-N induced oxidative stress in human ovarian cancer IGROV1 cells. Mol BioSyst. 2015;11:3059-67.

47. Grassi ML, Palma C de S, Thomé CH, Lanfredi GP, Poersch A, Faça VM. Proteomic analysis of ovarian cancer cells during epithelial-mesenchymal transition (EMT) induced by epidermal growth factor (EGF) reveals mechanisms of cell cycle control. J Proteome. 2017;151:2-11.

48. Westbrook JA, Noirel J, Brown JE, Wright PC, Evans CA. Quantitation with chemical tagging reagents in biomarker studies. PROTEOMICS - Clin Appl. 2015:9:295-300.

49. Kristjansdottir B, Levan K, Partheen K, Carlsohn E, Sundfeldt K. Potential tumor biomarkers identified in ovarian cyst fluid by quantitative proteomic analysis, iTRAQ. Clin Proteomics. 2013;10:4

50. Wang L-N, Tong S-W, Hu H-D, Ye F, Li S-L, Ren H, et al. Quantitative proteome analysis of ovarian cancer tissues using a iTRAQ approach. J Cell Biochem. 2012;113:3762-72.

51. Wang L, Chen S, Zhang M, Li N, Chen Y, Su W, et al. Legumain: a biomarker for diagnosis and prognosis of human ovarian cancer. J Cell Biochem. 2012; 113:2679-86.

52. Russell MR, Walker MJ, Williamson AJK, Gentry-Maharaj A, Ryan A, Kalsi J, et al. Protein Z: a putative novel biomarker for early detection of ovarian cancer. Int J Cancer. 2016;138:2984-92.

53. Waldemarson S, Krogh M, Alaiya A, Kirik U, Schedvins K, Auer G, et al. Protein expression changes in ovarian Cancer during the transition from benign to malignant. J Proteome Res. 2012;11:2876-89.

54. Hiramatsu K, Yoshino K, Serada S, Yoshihara K, Hori Y, Fujimoto M, et al. Similar protein expression profiles of ovarian and endometrial high-grade serous carcinomas. Br J Cancer. 2016;114:554-61.

55. Sinclair J, Metodieva G, Dafou D, Gayther SA, Timms JF. Profiling signatures of ovarian cancer tumour suppression using 2D-DIGE and 2D-LC-MS/MS with tandem mass tagging. J Proteome. 2011;74:451-65.

56. Nepomuceno Al, Shao H, Jing K, Ma Y, Petitte JN, Idowu MO, et al. In-depth LC-MS/MS analysis of the chicken ovarian cancer proteome reveals conserved and novel differentially regulated proteins in humans. Anal Bioanal Chem. 2015:407:6851-63.

57. Wegdam W, Argmann CA, Kramer G, Vissers JP, Buist MR, Kenter GG, et al. Label-free LC-MSe in tissue and serum reveals protein networks underlying differences between benign and malignant serous ovarian tumors. Rota R, editor. PLoS One. 2014;e108046:9.

58. Langley SR, Mayr M. Comparative analysis of statistical methods used for detecting differential expression in label-free mass spectrometry proteomics. J Proteome. 2015:129:83-92.

59. Banazadeh A, Veillon L, Wooding KM, Zabet-moghaddam M, Mechref Y. Recent advances in mass spectrometric analysis of glycoproteins. Electrophoresis. 2017;38:162-89. 
60. Mechref Y, Hu Y, Garcia A, Zhou S, Desantos-Garcia JL, Hussein A. Defining putative glycan cancer biomarkers by MS. Bioanalysis. 2012;4:2457-69.

61. Tousi F, Hancock WS, Hincapie M. Technologies and strategies for glycoproteomics and glycomics and their application to clinical biomarker research. Anal Methods. 2011;3:20-32.

62. Kim K, Ruhaak LR, Nguyen UT, Taylor SL, Dimapasoc L, Williams C, et al. Evaluation of glycomic profiling as a diagnostic biomarker for epithelial ovarian cancer. Cancer Epidemiol Biomark Prev. 2014;23:611-21.

63. Abbott KL, Lim J-M, Wells L, Benigno BB, McDonald JF, Pierce M. Identification of candidate biomarkers with cancer-specific glycosylation in the tissue and serum of endometrioid ovarian cancer patients by glycoproteomic analysis. Proteomics. 2010;10:470-81.

64. Shetty V, Hafner J, Shah P, Nickens Z, Philip R. Investigation of ovarian cancer associated sialylation changes in $\mathrm{N}$-linked glycopeptides by quantitative proteomics. Clin Proteomics. 2012;9:10.

65. Kuzmanov U, Musrap N, Kosanam H, Smith CR, Batruch I, Dimitromanolakis A, et al. Glycoproteomic identification of potential glycoprotein biomarkers in ovarian cancer proximal fluids. Clin Chem Lab Med. 2013:51.

66. Saldova R, Struwe WB, Wynne K, Elia G, Duffy MJ, Rudd PM. Exploring the glycosylation of serum CA125. Int J Mol Sci. 2013;14:15636-54.

67. Liau B, Tan B, Teo G, Zhang P, Choo A, Rudd PM. Shotgun Glycomics identifies tumor-associated glycan ligands bound by an ovarian carcinomaspecific monoclonal antibody. Sci Rep. 2017;7:14489.

68. Maes E, Tirez K, Baggerman G, Valkenborg D, Schoofs L, Encinar JR, et al. The use of elemental mass spectrometry in phosphoproteomic applications. Mass Spectrom Rev. 2016;35:350-60.

69. Toss A, De Matteis E, Rossi E, Casa L, lannone A, Federico M, et al. Ovarian Cancer: can proteomics give new insights for therapy and diagnosis? Int J Mol Sci. 2013;14:8271-90

70. Harsha HC, Pandey A. Phosphoproteomics in cancer. Mol Oncol. 2010;4: 482-95.

71. Francavilla C, Lupia M, Tsafou K, Villa A, Kowalczyk K, Rakownikow JersieChristensen $\mathrm{R}$, et al. Phosphoproteomics of primary cells reveals Druggable kinase signatures in ovarian Cancer. Cell Rep. 2017:18:3242-56.

72. Crutchfield CA, Thomas SN, Sokoll L, Chan DW. Advances in mass spectrometry-based clinical biomarker discovery. Clin Proteomics. 2016;13:1.

73. Mann M, Ong SE, Grønborg M, Steen H, Jensen ON, Pandey A. Analysis of protein phosphorylation using mass spectrometry: deciphering the phosphoproteome. Trends Biotechnol. 2002;20:261-8.

74. Gustafsson JOR, Oehler MK, Ruszkiewicz A, McColl SR, Hoffmann P. MALD imaging mass spectrometry (MALDI-IMS)-application of spatial proteomics for ovarian cancer classification and diagnosis. Int J Mol Sci. 2011;12:773-94.

75. Zhu Y, Wu R, Sangha N, Yoo C, Cho KR, Shedden KA, et al. Classifications of ovarian cancer tissues by proteomic patterns. Proteomics. 2006;6:5846-56.

76. Kim H, Wu R, Cho KR, Thomas DG, Gossner G, Liu JR, et al. Comparative proteomic analysis of low stage and high stage endometrioid ovarian adenocarcinomas. Proteomics Clin Appl. 2008;2:571-84.

77. Schwamborn K, Kriegsmann M, Weichert W. MALDI imaging mass spectrometry — from bench to bedside. Biochim Biophys Acta - Proteins Proteomics. 2016.

78. Lemaire R, Ait Menguellet S, Stauber J, Marchaudon V, Lucot J-P, Collinet P, et al. Specific MALDI imaging and profiling for biomarker hunting and validation: fragment of the $11 \mathrm{~S}$ proteasome activator complex, Reg alpha fragment, is a new potential ovary Cancer biomarker. J Proteome Res. 2007; 6:4127-34.

79. McDonnell LA, Corthals GL, Willems SM, van Remoortere A, RJM v Z, Deelder AM. Peptide and protein imaging mass spectrometry in cancer research. J Proteome. 2010;73:1921-44.

80. Kang S, Shim HS, Lee JS, Kim DS, Kim HY, Hong SH, et al. Molecular proteomics imaging of tumor interfaces by mass spectrometry. J Proteome Res. 2010;9:1157-64.

81. Everest-Dass AV, Briggs MT, Kaur G, Oehler MK, Hoffmann P, Packer NH. $\mathrm{N}$-glycan MALDI imaging mass spectrometry on formalin-fixed paraffinembedded tissue enables the delineation of ovarian Cancer tissues. Mol Cell Proteomics. 2016;15:3003-16.

82. Delcourt V, Franck J, Leblanc E, Narducci F, Robin Y-M, Gimeno J-P, et al Combined mass spectrometry imaging and top-down microproteomics reveals evidence of a hidden proteome in ovarian Cancer. EBioMedicine. 2017:21:55-64

83. Jayson GC, Kohn EC, Kitchener HC, Ledermann JA. Ovarian cancer. Lancet 2014;384:1376-88
84. O'Toole S, O'Leary J. Ovarian Cancer Chemoresistance. Encycl Cancer. 2011:2674-6.

85. Deng J, Wang L, Ni J, Beretov J, Wasinger V, Wu D, et al. Proteomics discovery of chemoresistant biomarkers for ovarian cancer therapy. Expert Rev Proteomics. 2016;13:905-15.

86. Agarwal R, Kaye SB. Ovarian cancer: strategies for overcoming resistance to chemotherapy. Nat Rev Cancer. 2003;3:502-16.

87. Yan X, Pan L, Yuan Y, Lang J, Mao N. Identification of platinum-resistance associated proteins through proteomic analysis of human ovarian Cancer cells and their platinum-resistant sublines. J Proteome Res. 2007;6:772-80.

88. Dai Z, Yin J, He H, Li W, Hou C, Qian X, et al. Mitochondrial comparative proteomics of human ovarian cancer cells and their platinum-resistant sublines. Proteomics. 2010;10:3789-99.

89. Cicchillitti L, Di Michele M, Urbani A, Ferlini C, Donat MB, Scambia G, et al. Comparative proteomic analysis of paclitaxel sensitive A2780 epithelial ovarian cancer cell line and its resistant counterpart A2780TC1 by 2D-DIGE: the role of ERp57. J Proteome Res. 2009;8:1902-12.

90. Lee DH, Chung K, Song J-A, Kim T, Kang H, Huh JH, et al. Proteomic identification of paclitaxel-resistance associated hnRNP A2 and GDI 2 proteins in human ovarian Cancer cells. J Proteome Res. 2010;9:5668-76.

91. Di Michele M, Marcone S, Cicchillitti L, Della Corte A, Ferlini C, Scambia G, et al. Glycoproteomics of paclitaxel resistance in human epithelial ovarian cancer cell lines: towards the identification of putative biomarkers. J Proteome. 2010;73:879-98.

92. Chappell NP, Teng P, Hood BL, Wang G, Darcy KM, Hamilton CA, et al. Mitochondrial proteomic analysis of cisplatin resistance in ovarian Cancer. J Proteome Res. 2012;11:4605-14

93. Zhang S-F, Wang X-Y, Fu Z-Q, Peng Q-H, Zhang J-Y, Ye F, et al. TXNDC17 promotes paclitaxel resistance via inducing autophagy in ovarian cancer. Autophagy. 2015;11:225-38.

94. Chen X, Wei S, Ma Y, Lu J, Niu G, Xue Y, et al. Quantitative proteomics analysis identifies mitochondria as therapeutic targets of multidrugresistance in ovarian cancer. Theranostics. 2014:4:1164-75.

95. Stewart JJ, White JT, Yan X, Collins S, Drescher CW, Urban ND, et al. Proteins associated with cisplatin resistance in ovarian Cancer cells identified by quantitative proteomic technology and integrated with mRNA expression levels. Mol Cell Proteomics. 2006:5:433-43.

96. Pan S, Cheng L, White JT, Lu W, Utleg AG, Yan X, et al. Quantitative proteomics analysis integrated with microarray data reveals that extracellular matrix proteins, catenins, and P53 binding protein 1 are important for chemotherapy response in ovarian cancers. Omi A J Integr Biol. 2009;13:345-54.

97. Li S-L, Ye F, Cai W-J, Hu H-D, Hu P, Ren H, et al. Quantitative proteome analysis of multidrug resistance in human ovarian cancer cell line. J Cell Biochem. 2010;109:n/a-n/a

98. Shetty V, Nickens Z, Testa J, Hafner J, Sinnathamby G, Philip R. Quantitative immunoproteomics analysis reveals novel MHC class I presented peptides in cisplatin-resistant ovarian cancer cells. J Proteome. 2012;75:3270-90.

99. Yu K-H, Levine DA, Zhang H, Chan DW, Zhang Z, Snyder M. Predicting ovarian Cancer patients' clinical response to platinum-based chemotherapy by their tumor proteomic signatures. J Proteome Res. 2016;15:2455-65.

100. Maes E, Mertens I, Valkenborg D, Pauwels P, Rolfo C, Baggerman G. Proteomics in cancer research: are we ready for clinical practice? Crit Rev Oncol Hematol. 2015;96:437-48.

101. Greco V, Piras C, Pieroni L, Ronci M, Putignani L, Roncada P, et al. Applications of MALDI-TOF mass spectrometry in clinical proteomics. Expert Rev Proteomics. 2018:14789450.2018.1505510.

102. Levitsky LI, Ivanov MV, Lobas AA, Gorshkov MV. Unbiased false discovery rate estimation for shotgun proteomics based on the target-decoy approach. J Proteome Res. 2017;16:393-7. 